Original article

\title{
SARS-CoV2 IgG antibody: Seroprevalence among health care workers
}

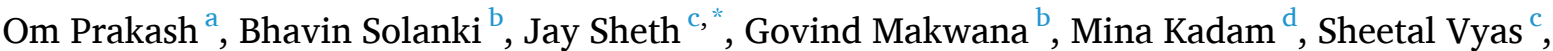 Aparajita Shukla ${ }^{\mathrm{e}}$, Jayshri Pethani ${ }^{\mathrm{f}}$, Hemant Tiwari ${ }^{\mathrm{e}}$}

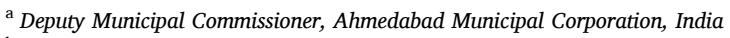

${ }^{\mathrm{b}}$ Health Department, Ahmedabad Municipal Corporation, India

${ }^{\mathrm{c}}$ Department of Community Medicine, AMC MET Medical College, India

${ }^{\mathrm{d}}$ Department of Microbiology, AMC MET Medical College, India

${ }^{\mathrm{e}}$ Department of Community Medicine, NHL Municipal Medical College, India

${ }^{\mathrm{f}}$ Department of Microbiology, NHL Municipal Medical College, India

\section{A R T I C L E I N F O}

\section{Keywords:}

IgG antibodies

Covid-19

Health care workers

Sero-surveillance

Immunity

SARS-COV2

\begin{abstract}
A B S T R A C T
Background: Health Care Workers (HCWs) are at higher risk for Covid19. Sero-surveillance among HCWs using IgG antibodies can add further value to the scientific findings.

Objectives: To estimate seropositivity among HCWs and to correlate it with various factors affecting seropositivity.

Methods: Population based large scale sero-surveillance among HCWs was carried out during second half of August'20 in Ahmedabad using "Covid-Kavach” IgG Antibody Detection ELISA kits. Seropositivity among HCWs was estimated and compared with various demographic \& other factors to understand their infection \& immunity status. Proportions and Z-test were used as appropriate.

Results: As on August'20, Seropositivity among HCWs from Ahmedabad is $23.65 \%$ (95\% Confidence Interval $21.70-25.73 \%$ ). Seropositivity of $25.98 \%$ (95\%CI 23.47-28.66) among female HCWs is significantly higher than 19.48\% (95\%CI 16.53-22.80) among male HCWs. The zone wise positivity among HCWs closely correlate with cases reported from the respective zone. The sero-positivity among HCWs from the earliest and worst affected zones have lower level of seropositivity as compared to the zones affected recently. This might be pointing towards the fact that the IgG Antibodies may not be long lasting.

Conclusion: As on August 2020, the seropositivity of 23.65\% in HCWs indicate high level of disease transmission and higher risk of infection among HCWs in Ahmedabad. The seropositivity is significantly higher among female HCWs. Zone wise seropositivity, closely correlate with the reported cases from the respective zone. Their comparison also indicates the possibility of reducing IgG seropositivity, which necessitates further in-depth scientific research to generate greater scientific evidences.
\end{abstract}

\section{Introduction}

Covid19, the disease caused by SARS-CoV2, spread across the world during $2020 .{ }^{1}$ In view of the asymptomatic infection of Covid19, we cannot rely on the data of identified cases as those who exhibit symptoms are more likely to get tested than asymptomatic individuals. ${ }^{2}$ Sero-surveillance uncover the asymptomatic, subclinical infection and helps in understanding the disease transmission dynamics in a better way for planning an appropriate public health response. ${ }^{3}$ Multiple sero-surveillance studies conducted during the pandemic have focused on antibodies against SARS-CoV2 in the general population. ${ }^{4,5}$

HCWs are exposed to suspected/confirmed cases and their contacts as part of their job-profile. This occupational exposure put them at a higher risk of infection. ${ }^{6}$ Frontline HCWs working in hospitals had a significantly higher seroprevalence than HCW in other settings. ${ }^{7}$ None the less, HCWs working in the field, both from public sector and private sector, are still at higher risk of infection. These HCWs could be a source of infection as they can transmit the infection. ${ }^{8}$ Thus, seroprevalence

\footnotetext{
* Corresponding author.

E-mail addresses: dromprakash2006@gmail.com (O. Prakash), bhavinpsm@gmail.com (B. Solanki), jayksheth@yahoo.com (J. Sheth), gtmakwana@yahoo.com

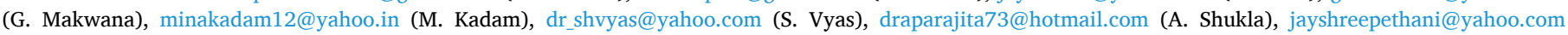
(J. Pethani), tiwarihemant1@rediffmail.com (H. Tiwari).
} 
among field level HCWs is a crucial indicator giving better scientific insight into disease situation.

Ahmedabad city with approximately 7 million people was one of the earliest cities to witness the high number of cases during the initial months of the pandemic in India. We carried out a population based sero-surveillance during the second half of August'20. In this serosurveillance, apart from the general population, HCWs were also included as an additional category along with cases \& contacts of cases. This article focuses and describes only the "HCWs" component of the sero-surveillance. Keeping estimation of seroprevalence among HCWs as our primary objective, we also checked the correlation of seropositivity with various demographic and other factors affecting their immunity.

\section{Materials and methods}

Indian Council of Medical Research (ICMR) had issued directives for conducting IgG Antibody based ELISA test for sero-surveys to monitor the pandemic, understand its progression and to take appropriate corrective public health measures. Ahmedabad Municipal Corporation (AMC), from the state of Gujarat, INDIA, planned and conducted a serosurvey using population based stratified sampling during second half of August 2020. Result of the earlier sero-survey in general population of Ahmedabad was used for the purpose of calculating the sample size for the present study. Ward/Urban Primary Health Centre (UPHC) wise required minimum sample size for the general population category was determined based on population proportion with $95 \%$ confidence level with $1 \%$ margin of error. Along with the general population category, HCWs were also enrolled separately as an additional category along with cases and contact of cases. The sample size for the "HCWs" category was decided as atleast $10 \%$ of general population sample target. This sample size was calculated for each ward/UPHC, and thus, the sample size for HCWs was also based on population proportion.

"Covid-Kavach" (Anti-SARS CoV2 IgG Antibody Detection capture ELISA) kits developed and manufactured by Zydus Diagnostics \& validated by National Institute of Virology, Pune, India were used for the purpose of this study. ICMR has permitted its use for sero-surveillance of SARS-CoV2 as its results are quite reliable with sensitivity of $92.37 \%$ and specificity of $97.9 \%$ as per the validation reports. ${ }^{9}$ The manufacturer reported no cross-reactivity with other viruses in the serum from real-time RTPCR confirmed patients of various other infections. Testing procedures were followed as per the manufacturer's instructions.

There are 75 UPHCs within 48 wards, across 7 zones in Ahmedabad. There are various categories of health workers posted at these UPHCs. There are other health care workers from the field area of UPHCs, who are working at private dispensaries, hospitals, other health care setup etc. All such HCWs were eligible to get enrolled in the study in the HCW category. All UPHCs tried to enroll all such eligible under the Health Care Worker category for the purpose of serosurveillance. Thus, the sample included a mix of doctors, nurses, paramedical, field level health care workers etc - all directly or indirectly associated with the health care field. All these health care workers were enrolled without any bias of designation, work setting or affiliations, since estimation of seroprevalence among HCWs was the primary objective.

Methodology in brief

Study site: Ahmedabad City of Gujarat, India.

Study period: Second half of August 2020.

Study design: Cross sectional sero-surveillance.

Study category: Health Care Workers.

Sampling technique: General population based stratified sampling.

Sample size: atleast $10 \%$ of the general population sample, which is calculated based on population proportion.

Testing Kit: Covid-Kavach (Anti-SARS CoV2 IgG Antibody Detection capture ELISA).

The study was carried out after the approval of ethics committee of AMC MET Medical College. Written informed consent was taken for the purpose of the study from all the participants before enrollment. Strict confidentiality was ensured at all levels. For the purpose of testing and standardization, only those laboratories with national level accreditation and state of the art facilities and equipment were approved for testing the samples. To reduce the sample rejection rate, SST-Gel Vacutee were used for the collection of blood samples. Microsoft Excel and Epi-Info was used for the purpose of data management. The crude positivity in HCWs was considered as an indicator for the current level of immunity among field level HCWs. In-depth analysis of the data was carried out with focus on comparing seropositivity among HCWs with various demographic and other factors. Simple proportions and appropriate statistical tests were used wherever required. In this article, we have tried to focus only on the analysis of seroprevalence among HCWs. The comparison of seroprevalence among HCWs with the seroprevalence among general population and other categories have been addressed in other articles and not discussed at stretch in the present study. We herewith share the findings of our results for the detailed insight by the scientific community.

\subsection{Limitations}

We have collected limited demographic details of the enrolled HCWs so that only limited analysis on serosurveillance result was possible. The limitations of the testing kit "Covid-Kavach" automatically applies to the findings of our study.

\section{Results}

A total of 1710 (1098 female, 612 male) samples from HCWs were collected out of which 2 samples were rejected, one each from both the sex groups. Results were thus available for 1708 samples. From these results $1288(75.41 \%)$ were negative and $16(0.94 \%)$ had indeterminate results. Thus, a total of 404 results were positive for the IgG antibodies against SARS-CoV2 giving an overall positivity of $23.65 \%$ [95\% Confidence Interval (CI) 21.70-25.73\%].

Detailed analysis of 1708 HCWs (Table- 1 ) show that there were 1097 female and 611 male HCWs for whom results were available. A total of 285 samples were positive among female giving a positivity rate of $25.98 \%$ [95\%CI $23.47-28.66 \%$ ] whereas 119 samples were positive among male giving a positivity of $19.48 \%$ [ $95 \%$ CI $16.53-22.80 \%$ ]. The percentage positivity is higher among female HCWs as compared to the male HCWs and the difference is statistically significant $(\mathrm{Z}=3.03, \mathrm{P}=$ 0.002).

The age distribution of the HCWs typically follows age-heaping bias (data not shown, only grouped data shown in Table- 1) as the age of the enrolled HCWs were approximate as replied by them and not verified with any official document. The age of the HCWs ranged from 16 to 82 years with a mode of 25 , median of 33 and an average of $34.84 \pm 10.64$ years. Among the sample, the mean age of females was $36.21 \pm 10.29$ years whereas the mean age of males is $32.38 \pm 10.83$ years. Considering the sero-positive HCWs, the mean age for females was $37.25 \pm$ 10.19 years where as that of male is $31.55 \pm 10.31$ years.

The age group wise analysis of seropositivity among HCWs (Fig. 1) shows that the lowest seropositivity is for the 10-19 years age group $(16.67 \%)$ and the highest seropositivity is for 50-59 years age group (27.22\%). The linear trendline shows increasing trend from around $20 \%-25 \%$ as the age-group increases from $10-19$ to $60-69$ years. When the same comparison of age-group and sero-positivity is done for both the sex groups (Fig. 2) it shows that female HCWs have higher seropositivity for most of the age groups. The linear trendline when plotted for both the sex groups shows that while female HCWs have decreasing seropositivity as the age group increases, male HCWs have increasing seropositivity with increase in the age group.

The zone wise analysis of total tests and positive tests when compared to calculate percent positivity shows that the positivity in various zones varies widely. The zone wise positivity ranges from $10.14 \%$ to $29.72 \%$. The zone wise positivity shows that the East Zone 
Table-1

Analysis of Covid19 sero-survey positivity in HCWs.

\begin{tabular}{|c|c|c|c|c|c|c|c|c|c|c|}
\hline & \multicolumn{3}{|l|}{ Female } & \multicolumn{3}{|l|}{ Male } & \multicolumn{3}{|l|}{ Total } & \multirow[b]{2}{*}{$95 \%$ Confidence Interval } \\
\hline & Results & Positive & \% Positivity & Results & Positive & \% Positivity & Results & Positive & \% Positivity & \\
\hline Total & 1097 & 285 & 25.98 & 611 & 119 & 19.48 & 1708 & 404 & 23.65 & $21.67-25.70$ \\
\hline \multicolumn{11}{|c|}{ Age group } \\
\hline $10-19$ & 6 & 2 & 33.33 & 18 & 2 & 11.11 & 24 & 4 & 16.67 & $04.74-37.38$ \\
\hline $20-29$ & 327 & 67 & 20.49 & 294 & 64 & 21.77 & 621 & 131 & 21.10 & $18.07-24.48$ \\
\hline $30-39$ & 342 & 102 & 29.82 & 174 & 31 & 17.82 & 516 & 133 & 25.78 & 22.19-29.72 \\
\hline $40-49$ & 291 & 73 & 25.09 & 59 & 11 & 18.64 & 350 & 84 & 24.00 & $19.82-28.74$ \\
\hline $50-59$ & 118 & 38 & 32.20 & 51 & 8 & 15.69 & 169 & 46 & 27.22 & $20.67-34.59$ \\
\hline $60-69$ & 11 & 2 & 18.18 & 13 & 3 & 23.08 & 24 & 5 & 20.83 & $07.13-42.15$ \\
\hline $70-79^{a}$ & 1 & 0 & 0.00 & 2 & 0 & 0.00 & 3 & 0 & 0.00 & $00.00-00.00$ \\
\hline $80-89^{a}$ & 1 & 1 & 100 & 0 & 0 & 0.00 & 1 & 1 & 100 & $02.50-100.0$ \\
\hline \multicolumn{11}{|l|}{ Zone } \\
\hline $\mathrm{CZ}$ & 109 & 25 & 22.94 & 59 & 13 & 22.03 & 168 & 38 & 22.62 & $16.53-29.70$ \\
\hline EZ & 269 & 83 & 30.86 & 54 & 13 & 24.07 & 323 & 96 & 29.72 & $25.00-34.92$ \\
\hline NWZ & 86 & 10 & 11.63 & 62 & 5 & 8.06 & 148 & 15 & 10.14 & 05.78-16.17 \\
\hline $\mathrm{NZ}$ & 174 & 52 & 29.89 & 97 & 26 & 26.80 & 271 & 78 & 28.78 & $23.47-34.57$ \\
\hline SWZ & 79 & 16 & 20.25 & 69 & 4 & 5.80 & 148 & 20 & 13.51 & $08.45-20.10$ \\
\hline SZ & 210 & 55 & 26.19 & 122 & 22 & 18.03 & 332 & 77 & 23.19 & $18.97-28.02$ \\
\hline WZ & 170 & 44 & 25.88 & 148 & 36 & 24.32 & 318 & 80 & 25.16 & $20.70-30.20$ \\
\hline
\end{tabular}

${ }^{a}$ Due to less numbers in sample, data not reflected in Figure- 2 \& Figure- 3

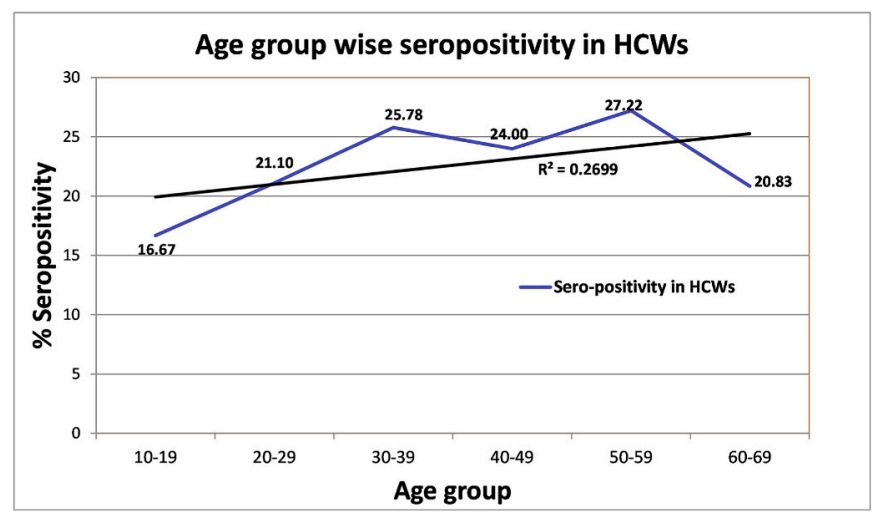

Figure-1. Age group wise seropositivity in HCWs.



Figure-2. Age group and sex wise seropositivity in HCWs.

(EZ-29.72\%) had the highest seropositivity followed by North Zone (NZ-28.78\%) whereas North West Zone (NWZ-10.14\%) and the South West Zone (SWZ-13.51\%) were the zones with lowest seropositivity.

There is close correlation while comparing the reported covid19 cases and seropositivity in HCWs for each zone (Fig. 3). The zones with low number of reported cases (as on $31 \mathrm{Jul} \& 15$ Aug '20) i.e., NWZ \& SWZ, have lower seropositivity among HCWs whereas other zones with higher number of reported cases (either during recent past or at the beginning of the pandemic) have higher seropositivity among HCWs.

\section{Discussion}

Although the scientific community is aware of the general immune response after any viral infection, the information about immune response during and after covid19 infection is still largely evolving. ${ }^{10}$ Multiple sero-surveillance studies which have focused on antibodies against SARS-CoV2 among various categories have been found to be extremely useful in understanding the progress of the pandemic. Scientific studies have recommended continued surveillance through seroprevalence studies to estimate and monitor the growing burden of Covid-19. ${ }^{11}$ The seroprevalence varies markedly due to a variety of factors. ${ }^{12}$ For the same reason, understanding the factors affecting immunity is extremely crucial while interpreting the results of the serosurveillance.

The present study focuses on the seropositivity among HCWs from Ahmedabad city of Gujarat, India. Seropositive HCWs are those who had been infected with SARS-CoV2 with/without symptom and who have developed IgG antibodies as a result of their clinical/subclinical infection, which is most likely to be due to an occupational exposure. Seroprevalence among HCWs is an important indicator as it gives an idea of communicability of the infection \& transmissibility of the infectious agent along with level of preventive/protective measures applied by the HCWs.

As per our results, as of August '20, the average seropositivity for IgG antibodies against SARS-CoV2 among HCWs from Ahmedabad is $23.65 \%$ [95\%CI 21.70-25.73\%]. As HCWs are aware of the transmission dynamics \& provided with appropriate personal protective equipment, one would expect them to have low infection rate and thus with lower seropositivity. However, within just 4-5 months of pandemic with $23.65 \%$ positivity, a large number of HCWs have acquired symptomatic/ asymptomatic infection and thus demonstrated antibodies. While comparing our study results with other studies among HCWs, the seroprevalence varies greatly from around $1 \%-24.4 \% .^{13,14}$ However, depending on the type of setting, work profile, exposure risk etc the seropositivity varies a lot. So, comparison and interpretation of seropositivity among HCWs must be extremely careful. Along with the seropositivity among HCWs (covered in this article), our study also measured seropositivity among general population. The seropositivity of $23.65 \%$ among HCWs, is quite close to the seropositivity among general population category (23.24\%). The higher seropositivity among HCWs, as compared to general population indicate widespread disease 


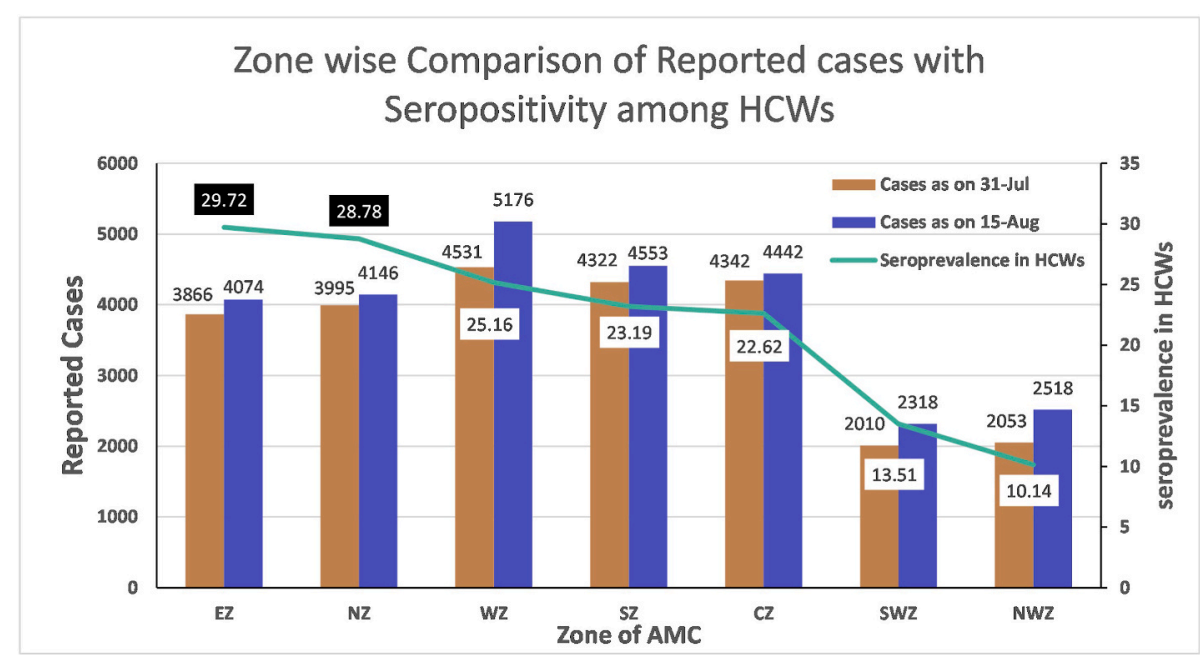

Figure-3. Zone wise comparison of cases and seropositivity in HCWs.

transmission and justifies the high level of infection during the initial months of the pandemic in Ahmedabad as compared to other cities of India as also reported by ICMR. ${ }^{15}$

Among the HCWs, females have higher positivity of $25.98 \%$ [95\%CI 23.47-28.66\%] as compared to male HCWs who demonstrated positivity of $19.48 \%$ [ $95 \%$ CI $16.53-22.80 \%$ ]. The significantly higher seropositivity among female HCWs as compared to male HCWs, need to be scientifically analyzed in detail to identify reasons for the same. This is in contrast to other studies where the sex-wise difference is statistically not significant. ${ }^{16}$

From the age analysis, the statistic of mode $<$ median $<$ mean typically shows skewed distribution. ${ }^{17}$ This indicate that our study sample of HCWs included more of young adult HCWs as compared to the middle aged \& elderly HCWs. Looking at the age-groups, 0-9 years age group is not applicable in HCWs. Only 4 HCWs above 70 years were enrolled and only 1 (from 8089 years age group) was sero-positive. So, we plotted the age-group wise analysis up to 60-69 years only, which covered most of the HCWs without any bias of the outliers. The age group wise analysis of seropositivity among HCWs (Fig. 1) shows that the seropositivity is seen in the range of $16.67 \%-27.22 \%$. The positivity is lower on both the extremes of the age groups, i.e. 10-19 and 60-69 years age groups. In both these age groups, the sample size is comparatively low. Moreover, health authorities at Ahmedabad have advised low risk work assignment to the $50+$ age groups particularly those HCWs with any of the known comorbid condition. So, in the HCWS with $50+$ age, there was reduced occupational exposure as compared to other HCWs, atleast in the public health sector. Even in the private health care sector, during the early months of the pandemic, elderly health care workers particularly with comorbidity were avoiding practice and direct clinical exposure. All the above details could possibly explain the low seropositivity in the extremes of the age groups, from whatever little sample that we have enrolled.

Seropositivity level is seen on higher side in the age groups representing young adults and middle aged HCWs. This may be related to their field work duty assigned to the health care workers in the public health sector. Even in the private sector, the young aged HCWs were more active in health care services and thus more at risk of having a contact and more likely to develop antibodies against SARS-CoV2 which reflected as seropositivity in the serosurveillance.

While checking the trend of seropositivity with a linear trendline, it shows increasing seropositivity with increasing age group among HCWs. We noted that the scientific research studies have documented that young adults are more likely to be asymptomatic, have milder symptoms and for shorter duration, if at all, as compared to the elderly people. ${ }^{18-21}$ It is also documented that percent seroconversion in asymptomatic cases is low. ${ }^{22}$ This phenomenon holds true not only for general population but also for HCWs and this may be the reason for higher sero-conversion among middle aged as compared to the young adults.

When the same comparison of age-group and sero-positivity is done for both the sex groups (Fig. 2) it shows that female HCWs have higher seropositivity for most of the age groups. The linear trendline when plotted for both the sex groups shows that female HCWs have decreasing seropositivity as the age group increases. On the other hand, male HCWs have increasing seropositivity with increase in the age group. This is a typical finding and in the absence of other details for the enrolled HCWs (designation, work setting or affiliations), we can only suggest that a further in-depth scientific study is required to find out the reasons for this difference.

HCWs were selected with convenience sampling from the UPHC field areas \& the sample size was based on population proportion. HCWs are at a higher risk of contracting infection ( $\&$ thus immunity) as the cases from their field-work area increases, and this holds true for public sector HCWs as well as Private sector HCWs, as both of them are involved in serving the local community. This is the reason why we compared the seropositivity among HCWs with the cases reported from their zone. So, although our sampling was population based \& independent of the cases reported from the zone, but still, the positivity in HCWs closely correlate with the cases reported from their zone, higher for those zones with high current or past cases. The zone wise positivity ranged from $10.14 \%$ to $29.72 \%$.

Scientific studies have documented that antibodies take some time to develop after an infection, approximately $1-3$ weeks, with an average 2 weeks (14 days). ${ }^{23,24}$ So, the rate of antibody positivity reflects the case scenario about 14 days prior to the study. Accordingly, Since, our study was carried out during second half of August'20, we took cases as on July end (around 2 weeks before the start of our study) \& 15th August (around 2 weeks before the end of our study, coinciding with the starting point of our study). Comparing the zone wise cases with the seropositivity among HCWs (Figure- 3), it is seen that the zones with comparatively low number of cases [North West Zone (NWZ) \& South West Zone (SWZ)] have low seropositivity among HCWs. The comparison also shows that inspite of having similar number of cases in a narrow range, the sero-positivity among HCWs from the earliest and worst affected zones [Central Zone (CZ), South Zone (SZ)] have lower level of seropositivity as compared to the zones affected recently [North Zone (NZ), East Zone (EZ)]. This might be pointing towards the fact that the IgG Antibodies may not be long lasting. Scientific studies have also documented declining level of antibodies, which vanishes over a period of time. ${ }^{25-27}$ This needs further research to cross verify with scientific evidences to prove this observation. 


\section{Conclusion}

As on August 2020, the seropositivity of $23.65 \%$ in HCWs indicate high level of disease transmission and higher risk of infection among HCWs in Ahmedabad. The seropositivity is significantly higher among female HCWs. The zone wise seropositivity, closely correlate with the reported cases from the respective zone. Their comparison also indicates the possibility of reducing IgG seropositivity, which necessitates further in-depth scientific research to generate stronger scientific evidences.

\section{Source(s) of support}

NIL.

\section{Presentation at a meeting}

NIL.

\section{Conflicting interest}

None Declared.

\section{Ethical approval}

Protocol was approved by the IEC of the AMC MET Medical College (DCGI Registration No: ECR/17/inst/Guj/2013/RR-20) with Letter No. "NIL" dated 17/8/2020.

\section{Acknowledgments}

We are extremely thankful to respected Dr. Rajiv Kumar Gupta, IAS (Additional Chief Secretary, Government of Gujarat) and Mr. Mukesh Kumar, IAS (Municipal Commissioner, Ahmedabad) for their whole hearted support. We acknowledge the full support from the field level health care workers (Corona warriors) for the purpose of this study. All the Zonal Deputy Health Officers, Deputy Health Officer (Epidemic), Assistant Health Officers and Medical officers of the UPHCs extended full support in conducting the sero-surveillance. We are thankful to all the medical and paramedical support staff posted at the laboratories for their contribution in timely testing of the samples with accuracy and quality. Finally, we are indebted to all the participants whose willingness and support has generated the much-desired data for the study.

\section{References}

1 Munster VJ, Koopmans M, Van Doremalen N, Van Riel D. PERSPECTIVE 692 A Novel Coronavirus Emerging in China A Novel Coronavirus Emerging in China-Key Questions for Impact Assessment. 2020.

2 Swaminathan A, Subramanian SV [cited 2021 Feb 12]. Reflections on Designing Population Surveys for COVID-19 Infection and Prevalence. vol. 42. GeroScience. Springer Science and Business Media Deutschland GmbH; 2020. https://doi.org/ 10.1007/s11357-020-00253-6, 1445-8. Available from:, [Internet].

3 Organization WH. Population-based Age-Stratified Seroepidemiological Investigation Protocol for COVID-19 Virus Infection. World Health Organization; 2020, 17 March 2020.

$4 \mathrm{Xu}$ X, Sun J, Nie S, et al. Seroprevalence of immunoglobulin M and G antibodies against SARS-CoV-2 in China [cited 2021 Feb 9] Nat Med [Internet]. 2020 Aug 1;26 (8). https://doi.org/10.1038/s41591-020-0949-6. Available from:, 1193-5.

5 Murhekar MV, Bhatnagar T, Selvaraju S, et al. Prevalence of SARS-CoV-2 infection in India: findings from the national serosurvey. May-June 2020 Indian J Med Res [Internet]. 2020;152:48-60 [cited $2021 \mathrm{Feb} 9$ ] http://www.ijmr.org.in/articles/ 2020/152/1/images/. Available from.

6 Grant J, Wilmore S, McCann N, et al. Seroprevalence of SARS-CoV-2 antibodies in healthcare workers at a London NHS Trust [cited 2021 Feb 12] Infect Control Hosp Epidemiol [Internet]. 2020;42(2). Available from https://pubmed.ncbi.nlm.nih.gov/ $32746953 /$.
7 Iversen K, Bundgaard H, Hasselbalch RB, et al. Risk of COVID-19 in health-care workers in Denmark: an observational cohort study. Lancet Infect Dis. 2020 Dec 1;20 (12):1401-1408.

8 Stubblefield WB, Talbot HK, Feldstein LR, et al. Seroprevalence of SARS-CoV-2 among frontline healthcare personnel during the first month of caring for patients with COVID-19-nashville, Tennessee [cited 2021 Feb 12] Clin Infect Dis [Internet]; 2020 Jun 6. Available from https://pubmed.ncbi.nlm.nih.gov/32628750/.

9 Sapkal G, Shete-Aich A, Jain R, et al. Development of indigenous IgG ELISA for the detection of anti-SARS-CoV-2 IgG [cited $2021 \mathrm{Feb} 4$ 4] Indian J Med Res [Internet]. 2020 May 1;151(5):444. Available from: http://www.ijmr.org.in/text.asp?2020 $/ 151 / 5 / 444 / 286007$.

10 Chirathaworn C, Sripramote M, Chalongviriyalert P, et al. SARS-CoV-2 RNA shedding in recovered COVID-19 cases and the presence of antibodies against SARSCoV-2 in recovered COVID-19 cases and close contacts. Thailand, April-June 2020 PLoS One [Internet]. 2020 Oct 1;15(10 October). Available from: https://pubmed. ncbi.nlm.nih.gov/33119712/ [cited 2021 Feb 9].

11 Lai CC, Wang JH, Hsueh PR. Population-based seroprevalence surveys of anti-SARSCoV-2 antibody: an up-to-date review [Internet]. Elsevier B.V. Int J Infect Dis. 2020; 101. https://doi.org/10.1016/j.ijid.2020.10.011 [cited 2021 Feb 12], 314-22. Available from:

12 Rostami A, Sepidarkish M, Leeflang MMG, et al. SARS-CoV-2 seroprevalence worldwide: a systematic review and meta-analysis [Internet]. Elsevier B.V. Clin Microbiol Infect. 2020. https://doi.org/10.1016/j.cmi.2020.10.020 [cited 2021 Feb 12]. Available from:, Vol. 0

13 Brant-Zawadzki M, Fridman D, Robinson PA, et al. SARS-CoV-2 antibody prevalence in health care workers: preliminary report of a single center study. PloS One. 2020;15 (11), e0240006. https://doi.org/10.1371/journal.pone.0240006.

14 Shields A, Faustini SE, Perez-Toledo M, et al. SARS-CoV-2 seroprevalence and asymptomatic viral carriage in healthcare workers: a cross-sectional study. Thorax 2020;75:1089-1094.

15 The Economic Times. Prevalence of Covid-19 infection, at 55\%, is the highest in Ahmedabad containment zones: Sources. Available: https://economictimes. indiatimes. com/industry/healthcare/biotech/healthcare/prevalence- at- 55highest- in- abad- containment- zones/articleshow/77073909.cms?from $=$ mdr.

16 Stringhini S, Wisniak A, Piumatti G, et al. Seroprevalence of anti-SARS-CoV-2 IgG antibodies in Geneva, Switzerland (SEROCoV-POP): a population-based study. Lancet. 2020 Aug 1;396(10247):313-319.

17 von Hippel PT. Mean, median, and skew: correcting a textbook rule [cited $2021 \mathrm{Feb}$ 12] J Stat Educ [Internet]. 2005 Jan;13(2). Available from https://www.tandfonline. com/doi/full/10.1080/10691898.2005.11910556.

18 Wang X, Guo X, Xin Q, et al. Neutralizing Antibodies Responses to SARS-CoV-2 in COVID-19 Inpatients and Convalescent Patients [Internet]. medRxiv. medRxiv; 2020. https://doi.org/10.1101/2020.04.15.20065623 [cited 2021 Feb 4], 2020.04.15.20065623. Available from:

19 Hu WT, Christina Howell J, Ozturk T, et al. Antibody Profiles According to Mild or Severe SARS-CoV-2 Infection [Internet]vol. 26. Atlanta, Georgia, USA: Emerging Infectious Diseases. Centers for Disease Control and Prevention (CDC); 2020. https:// pubmed.ncbi.nlm.nih.gov/32857691/, 2020 [cited 2021 Feb 4]. pp. 2974-8. Available from:.

20 Cervia C, Nilsson J, Zurbuchen Y, et al. Systemic and mucosal antibody responses specific to SARS-CoV-2 during mild versus severe COVID-19. J Allergy Clin Immunol. 2021 Feb 1;147(2):545-557.e9.

21 Nikolai LA, Meyer CG, Kremsner PG, Velavan TP. Asymptomatic SARS coronavirus 2 infection: invisible yet invincible [internet]. Elsevier B.V. Int J Infect Dis. 2020;100. https://doi.org/10.1016/j.ijid.2020.08.076 [cited 2021 Feb 10], 112-6. Available from

22 Wellinghausen N, Plonné D, Voss M, Ivanova R, Frodl R, Deininger S. SARS-CoV-2IgG response is different in COVID-19 outpatients and asymptomatic contact persons [cited 2021 Feb 10] J Clin Virol [Internet]. 2020 Sep 1;130:104542. Available from https://pubmed.ncbi.nlm.nih.gov/32707511/.

23 Tripathi S, Deshmukh V, Patil A, Tipathi JP. COVID 19 diagnostic multiplicity and its role in community surveillance and control [Internet] [cited $2021 \mathrm{Feb} 4$ ] Infezioni Med Le; 2020:18-28. Available from: https://pubmed.ncbi.nlm.nih.gov/32532934/.

24 Amanat F, Stadlbauer D, Strohmeier S, et al. A serological assay to detect SARS-CoV2 seroconversion in humans [cited $2021 \mathrm{Feb}$ 10] Nat Med [Internet]. $2020 \mathrm{Jul}$ 1;26 (7). https://doi.org/10.1038/s41591-020-0913-5. Available from, 1033-6.

25 Sethuraman N, Jeremiah SS, Ryo A. Interpreting diagnostic tests for SARS-CoV-2 [internet] [cited $2021 \mathrm{Feb} 10]$ JAMA - Journal of the American Medical Association. American Medical Association. 2020;323. Available from https://jamanetwork.com/, 2249-51.

26 Rosado J, Pelleau S, Cockram C, et al. Serological Signatures of SARS-CoV-2 Infection: Implications for Antibody-Based Diagnostics [Internet]. medRxiv. medRxiv; 2020. https://doi.org/10.1101/2020.05.07.20093963 [cited 2021 Feb 10]. p. 2020.05.07.20093963, Available from:.

27 Seow J, Graham C, Merrick B, et al. Longitudinal observation and decline of neutralizing antibody responses in the three months following SARS-CoV-2 infection in humans. Available from Nat Microbiol [Internet]. 2020 Dec 1;5(12):1598-1607. https://doi.org/10.1038/s41564-020-00813-8 [cited 2021 Feb 10]. 